\title{
Simple and convenient analytical formulas for studying the projectile motion in midair
}

\author{
Peter Chudinov*1, Vladimir Eltyshev $^{1}$, Yuri Barykin ${ }^{1}$ \\ ${ }^{1}$ Engineering Faculty, Perm State Agricultural Academy, Perm, Russian Federation
}

Received on May 01, 2017. Revised on July 03, 2017. Accepted on July 12, 2017.

\begin{abstract}
Here is studied a classic problem of the motion of a projectile thrown at an angle to the horizon. The air drag force is taken into account as the quadratic resistance law. An analytic approach is used for the investigation. Equations of the projectile motion are solved analytically. The basic functional dependencies of the problem are described by elementary functions. There is no need to study the problem numerically. The found analytical solutions are highly accurate over a wide range of parameters The motion of a baseball and a badminton shuttlecock are presented as examples.
\end{abstract}

Keywords: classic problem, projectile motion, quadratic drag force, analytical formulas.

\section{Introduction}

The problem of the motion of a projectile in midair arouses interest of authors as before [1-8]. The number of publications on this problem is very large. Together with the investigation of the problem by numerical methods, attempts are still being made to obtain the analytical solutions. Many such solutions of a particular type are obtained. They are valid for limited values of the physical parameters of the problem (for the linear law of the medium resistance at low speeds, for short travel times, for low, high and split angle trajectory regimes and others). For the construction of the analytical solutions various methods are used - both the traditional approaches [1], and the modern methods [2, 5]. All proposed approximate analytical solutions are rather complicated and inconvenient for educational purposes. In addition, many approximate solutions use special functions, for example, the Lambert $\mathrm{W}$ function. This is why the description of the projectile motion by means of a simple approximate analytical formulas under the quadratic air resistance is of great methodological and educational importance.

The purpose of the present work is to give a simple formulas for the construction of the trajectory of the projectile motion with quadratic air resistance. In this paper, one of the variants of approximation of the sought functions (the projectile coordinates) is realized. It allows to construct a trajectory of the projectile with the help of elementary functions without using numerical schemes. Following other authors, we call this approach the analytic approach. The conditions of applicability of the quadratic resistance law are deemed to be fulfilled, i.e. Reynolds number Re lies within $1 \times 10^{3}<\operatorname{Re}<2 \times 10^{5}$.

*Correspondence email address: chupet@mail.ru

\section{Equations of projectile motion}

We now state the formulation of the problem and the equations of the motion according to [7]. Suppose that the force of gravity affects the projectile together with the force of air resistance $\boldsymbol{R}$ (Fig. 1). Air resistance force is proportional to the square of the velocity of the projectile and is directed opposite the velocity vector. For the convenience of further calculations, the drag force will be written as $R=m g k V^{2}$. Here $m$ is the mass of the projectile, $g$ is the acceleration due to gravity, $k$ is the proportionality factor. Vector equation of the motion of the projectile has the form

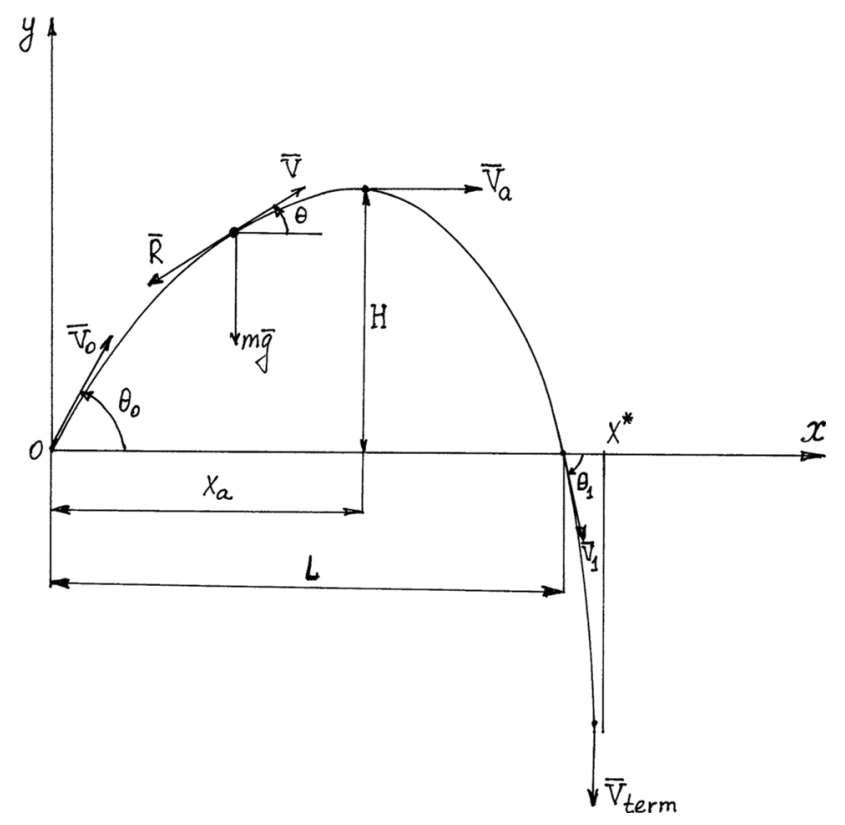

Figure 1: Basic motion parameters. 


$$
m \boldsymbol{w}=m \boldsymbol{g} \quad+\boldsymbol{R}
$$

where $\boldsymbol{w}$ - acceleration vector of the projectile. Differential equations of the motion, commonly used in ballistics, are as follows [9]

$$
\begin{aligned}
\frac{d V}{d t}=-g \sin \theta-g k V^{2} & , & \frac{d \theta}{d t}=-\frac{g \cos \theta}{V}, \\
\frac{d x}{d t}=V \cos \theta & , & \frac{d y}{d t}=V \sin \theta .
\end{aligned}
$$

Here $V$ is the velocity of the projectile, $\theta$ is the angle between the tangent to the trajectory of the projectile and the horizontal, $x, y$ are the Cartesian coordinates of the projectile,

$$
k=\frac{\rho_{a} c_{d} S}{2 m g}=\frac{1}{V_{\text {term }}^{2}}=\text { const }
$$

$\rho_{a}$ is the air density, $c_{d}$ is the drag factor for a sphere, $S$ is the cross-section area of the object, and $V_{\text {term }}$ is the terminal velocity The first two equations of the system (1) represent the projections of the vector equation of motion on the tangent and principal normal to the trajectory, the other two are kinematic relations connecting the projections of the velocity vector projectile on the axis $x, y$ with derivatives of the coordinates.

The well-known solution of system (1) consists of an explicit analytical dependence of the velocity on the slope angle of the trajectory and three quadratures

$$
\begin{aligned}
& V(\theta)=\frac{V_{0} \cos \theta_{0}}{\cos \theta \sqrt{1+k V_{0}^{2} \cos ^{2} \theta_{0}\left(f\left(\theta_{0}\right)-f(\theta)\right)}} \\
& f(\theta)=\frac{\sin \theta}{\cos ^{2} \theta}+\ln \tan \left(\frac{\theta}{2}+\frac{\pi}{4}\right) \\
& x=x_{0}-\frac{1}{g} \int_{\theta_{0}}^{\theta} V^{2} d \theta, y=y_{0}-\frac{1}{g} \int_{\theta_{0}}^{\theta} V^{2} \tan \theta d \theta \\
& t=t_{0}-\frac{1}{g} \int_{\theta_{0}}^{\theta} \frac{V}{\cos \theta} d \theta .
\end{aligned}
$$

Here $V_{0}$ and $\theta_{0}$ are the initial values of the velocity and of the slope of the trajectory respectively, $t_{0}$ is the initial value of the time, $x_{0}, y_{0}$ are the initial values of the coordinates of the projectile (usually accepted $\left.t_{0}=x_{0}=y_{0}=0\right)$. The derivation of the formulas (2) is shown in the well-known monograph [10]. The integrals on the right-hand sides of formulas (3) cannot be expressed in terms of elementary functions. Hence, to determine the variables $t, x$ and $y$ we must either integrate system (1) numerically or evaluate the definite integrals (3).

\section{Obtaining an analytical solution of the problem}

The analysis of the task shows, that equations (3) are not exactly integrable owing to the complicated nature of function (2). The odd function $f(\theta)$ is defined in the interval $-\frac{\pi}{2}<\theta<\frac{\pi}{2}$. Therefore, it can be assumed that a successful approximation of this function will make it possible to calculate analytically the definite integrals (3) with the required accuracy.

The Ref. [1] presents a simple approximation in the mathematical sense of a function $f(\theta)$ by a second-order polynomial of the following form (polynomial is with respect to a function $\tan \theta$ )

$$
f_{a}(\theta)=a_{1} \tan \theta+b_{1} \tan ^{2} \theta .
$$

An analysis of the problem shows that it is convenient to approximate the function $f(\theta)$ only by polynomials of the second or third degree. The first-order polynomial does not provide the required accuracy of the approximation. Polynomials of higher orders do not allow us to calculate the integrals (3) in elementary functions. The polynomial of the second order approximates the function $f(\theta)$ well only on a bounded interval $\left[0, \theta_{0}\right]$. Under the condition $\theta<0$, another approximation is required because the function $f(\theta)$ is odd. Therefore, the question of using a second-order polynomial for a given problem requires a separate study. As already noted, the function $f_{a}(\theta)$ well approximates the function $f(\theta)$ only on the limited interval $\left[0, \theta_{0}\right]$, since the function $f_{a}(\theta)$ contains an even term. Therefore, in the present paper we approximate the function $f(\theta)$ on the whole interval $-\frac{\pi}{2}<\theta<\frac{\pi}{2}$ with a function $f_{1}(\theta)$ of the following form

$$
f_{1}(\theta)=a_{1} \tan \theta+b_{1} \tan ^{3} \theta .
$$

The function $f_{1}(\theta)$ is formed by two odd functions. The coefficients $a_{1}$ and $b_{1}$ can be chosen in such a way as to smoothly connect the functions $f(\theta)$ and $f_{1}(\theta)$ to each other with the help of conditions

$$
f_{1}\left(\theta_{0}\right)=f\left(\theta_{0}\right), \quad f_{1}^{\prime}\left(\theta_{0}\right)=f^{\prime}\left(\theta_{0}\right) .
$$

From the conditions (4) we find

$$
\begin{array}{r}
a_{1}=\frac{1}{2 \cos \theta_{0}}+\frac{3 \ln \tan \left(\frac{\theta_{0}}{2}+\frac{\pi}{4}\right)}{2 \tan \theta_{0}}, \\
b_{1}=\frac{1}{2 \tan ^{2} \theta_{0}}\left(\frac{1}{\cos \theta_{0}}-\frac{\ln \tan \left(\frac{\theta_{0}}{2}+\frac{\pi}{4}\right)}{\tan \theta_{0}}\right) .
\end{array}
$$

Such a function $f_{1}(\theta)$ well approximates the function $f(\theta)$ throughout the whole interval of its definition for any values $\theta_{0}$. As an example, we give graphs of functions $f(\theta), f_{1}(\theta)$ in the interval $-80^{\circ} \leq \theta \leq 80^{\circ}$. Coefficients $a_{1}, b_{1}$ are calculated at a value $\theta_{0}=60^{\circ}$.

The solid curve in Figure 2 is a graph of the function $f(\theta)$, the dot curve is a graph of the function $f_{1}(\theta)$. The 

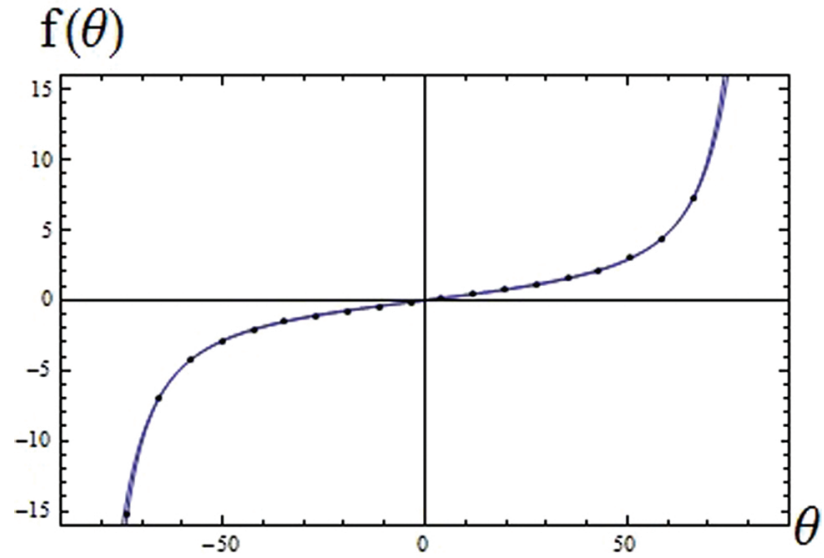

Figure 2: Approximation of the function $f(\theta)$.

graphs practically coincide. Hence, the function $f_{1}(\theta)$ can be used instead of the function $f(\theta)$ in calculating the integrals (3).

Now the quadratures (3) are integrated in elementary functions In calculating the integrals we take $t_{0}=x_{0}=$ $y_{0}=0$. We integrate the first of the integrals (3). For the coordinate $x$ we obtain:

$$
\begin{aligned}
& x=x_{0}-\frac{1}{g} \int_{\theta_{0}}^{\theta} V^{2} d \theta= \\
& =\left.\left(A_{1} \ln \left|\frac{(\tan \theta-b)^{2}}{\tan ^{2} \theta+b \tan \theta+c}\right|-A_{2} \arctan \left(\frac{2 \tan \theta+b}{\Delta}\right)\right)\right|_{\theta_{0}} ^{\theta} .
\end{aligned}
$$

Here we introduce the following notation:

$$
\begin{aligned}
& a=\frac{1}{k V_{0}^{2} \cos ^{2} \theta_{0}}+f\left(\theta_{0}\right), \\
& d_{0}=-\frac{a}{b_{1}}, \quad d_{1}=\frac{a_{1}}{b_{1}}, \quad p_{1}=\sqrt[3]{-\frac{d_{0}}{2}+\sqrt{\frac{d_{0}^{2}}{4}+\frac{d_{1}^{3}}{27}}} \\
& p_{2}=-\frac{d_{1}}{3 p_{1}}, \quad b=p_{1}+p_{2}, \quad c=d_{1}+b^{2} \\
& \Delta=\sqrt{\left|b^{2}-4 c\right|}, A_{1}=\frac{1}{2 g k b_{1}\left(c+b^{2}\right)}, A_{2}=\frac{6 b A_{1}}{\Delta} \\
& F_{1}(\theta)=A_{1} \ln \mid \frac{(\tan \theta-b)^{2}}{\tan ^{2} \theta+b \tan \theta+c \mid} \\
& A_{2} \arctan \left(\frac{2 \tan \theta+b}{\Delta}\right) .
\end{aligned}
$$

Thus, the dependence $x(\theta)$ has the following form:

$$
x(\theta)=F_{1}(\theta)-F_{1}\left(\theta_{0}\right) .
$$

We integrate the second of the integrals (3). For the coordinate $y$ we obtain:

$$
\begin{aligned}
& y=y_{0}-\frac{1}{g} \int_{\theta_{0}}^{\theta} V^{2} \tan \theta d \theta= \\
& =\left.\left(b F_{1}(\theta)+A_{3} \arctan \left(\frac{2 \tan \theta+b}{\Delta}\right)\right)\right|_{\theta_{0}} ^{\theta} .
\end{aligned}
$$

Here we introduce the following notation:

$$
\begin{aligned}
F_{2}(\theta) & =b F_{1}(\theta)+A_{3} \arctan \left(\frac{2 \tan \theta+b}{\Delta}\right), \\
A_{3} & =\frac{2}{g k b_{1} \Delta} .
\end{aligned}
$$

Thus, the dependence $y(\theta)$ has the following form:

$$
y(\theta)=F_{2}(\theta)-F_{2}\left(\theta_{0}\right) .
$$

Consequently, the basic functional dependencies of the problem $x(\theta), y(\theta)$ are written in terms of elementary functions. The main characteristics of the projectile's motion are the following ( Fig. 1):

$H$ - the maximum height of ascent of the projectile,

$T$ - motion time,

$L$ - flight range,

$x_{a}$ - the abscissa of the trajectory apex,

$t_{a}$ - the time of ascent,

$\theta_{1}$ - impact angle with respect to the horizontal . Using formulas $(5)$ - (6), we find:

$$
x_{a}=x(0), \quad H=y(0) .
$$

The third integral (3) is not taken in elementary functions. However, estimates for the parameters $T$ and $t_{a}$ can be made using the formulas of [6]. The angle of incidence of the projectile $\theta_{1}$ is determined from the condition $y\left(\theta_{1}\right)=0$. Then we have

$$
\begin{gathered}
L=x\left(\theta_{1}\right), \quad T=2 \sqrt{\frac{2 H}{g}}, \quad t_{a}=\frac{T-k H V_{a}}{2}, \\
V_{a}=\frac{V_{0} \cos \theta_{0}}{\sqrt{1+k V_{0}^{2} \cos ^{2} \theta_{0} f\left(\theta_{0}\right)}} .
\end{gathered}
$$

We note that formulas (5) - (6) also define the dependence $y=y(x)$ in a parametric way.

\section{The results of the calculations. Field of application of the obtained solutions}

Proposed formulas have a wide region of application. We introduce the notation $p=k V_{0}^{2}$. The dimensionless parameter $p$ has the following physical meaning - it is the ratio of air resistance to the weight of the projectile at the beginning of the movement. As calculations show, trajectory of the projectile $y=y(x)$ and the main characteristics of the motion $L, H, x_{a}$ have accuracy to within $1 \%$ for values of the launch angle and for the parameter $p$ within ranges

$$
0^{\circ}<\theta_{0}<90^{\circ}, 0<p \leq 60 .
$$

Figure 3 presents the results of plotting the projectile trajectories with the aid of formulas $(5)-(6)$ over a 
wide range of the change of the initial angle $\theta_{0}$ with the following values of the parameters

$$
\begin{gathered}
V_{0}=80 \mathrm{~m} / \mathrm{s}, k=0.000625 \mathrm{~s}^{2} / \mathrm{m}^{2}, \\
g=9.81 \mathrm{~m} / \mathrm{s}^{2}, p=4 .
\end{gathered}
$$

The used value of the parameter $k$ is the typical value of the baseball drag coefficient.

Analytical solutions are shown in Fig. 3 by dotted lines. The thick solid lines in Fig. 3 are obtained by numerical integration of system (1) with the aid of the 4-th order Runge-Kutta method. As it can be seen from Fig. 3, the analytical solutions (dotted lines) and a numerical solutions are the same.

Figure 4 represents the results of plotting the projectile trajectories with the aid of formulas $(5)-(6)$ over a wide range of the change of the initial velocity $V_{0}$. In this case the values of the parameter $p$ vary from 1 to 9 .

As an example of a specific calculation using formulas (5) - (6), we give the trajectory and the values of the basic parameters of the motion $L, H, T, x_{a}, t_{a}, \theta_{1}$ for shuttlecock in badminton. Of all the trajectories of sport projectiles, the trajectory of the shuttlecock has the

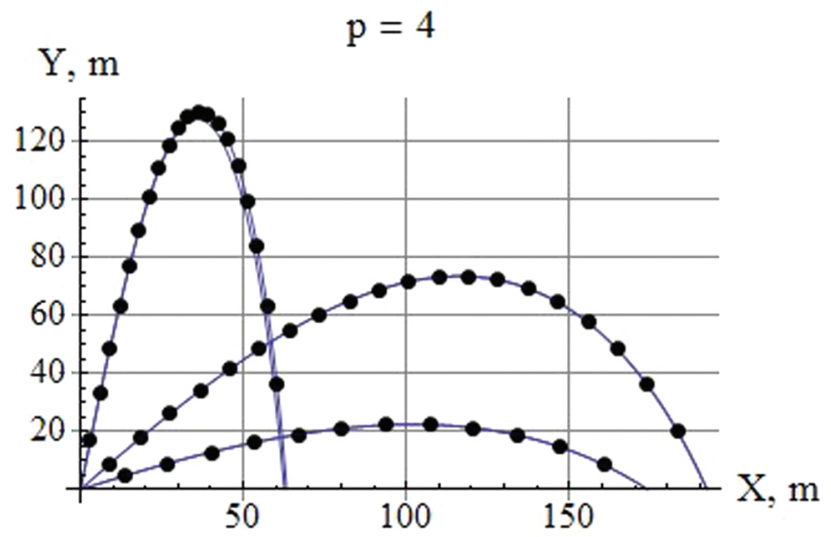

Figure 3: The graphs of the trajectory $y=y(x)$ at launching angles $\theta_{0}=20^{\circ}, 45^{\circ}, 80^{\circ}$.

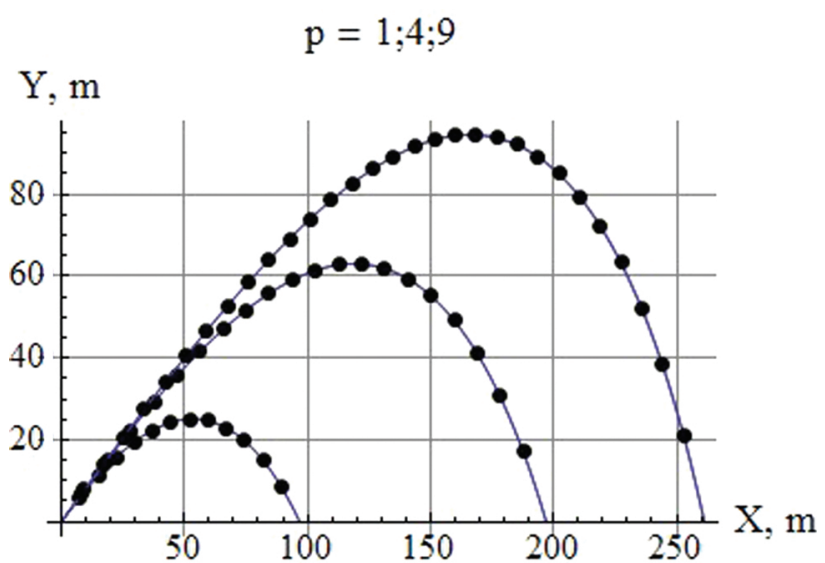

Figure 4: The graphs of the trajectory $y=y(x)$ under the initial conditions $\theta_{0}=40^{\circ}, V_{0}=40 \mathrm{~m} / \mathrm{s}, 80 \mathrm{~m} / \mathrm{s}, 120 \mathrm{~m} / \mathrm{s}$. greatest asymmetry. This is explained by the relatively large value of the drag coefficient $k$ and, accordingly, by the large values of the parameter $p$. Initial conditions of calculation are

$$
k=0.22 \mathrm{~s}^{2} / \mathrm{m}^{2}, \quad V_{0}=50 \mathrm{~m} / \mathrm{s}, \quad \theta_{0}=40^{\circ}, \quad p=55
$$

The trajectory of the shuttlecock is shown in Fig. 5. The second column of Table 1 contains range values calculated analytically with formulae $(7)-(8)$. The third column of Table 1 contains range values from the integration of the equations of system (1) The fourth column presents the error of the calculation of the parameter in the percentage. The error in calculating the basic motion parameters $L, H, x_{a}$ does not exceed $1 \%$. The parameters $T, t_{a}$ are determined in this example with low accuracy due to the large value of the parameter $p$. With smaller values of the parameter $p$, the values of $T, t_{a}$ are calculated rather accurately. For example, for $p=4$, the errors in calculating these parameters do not exceed $1.5 \%$.

Thus, a successful approximation of the function $f(\theta)$ made it possible to calculate the integrals (3) in elementary functions and to obtain a highly accurate analytical solution of the problem of the motion of the projectile in the air.

\section{Conclusions}

The proposed approach based on the use of analytic formulas makes it possible to simplify significantly a qualitative analysis of the motion of a projectile with the

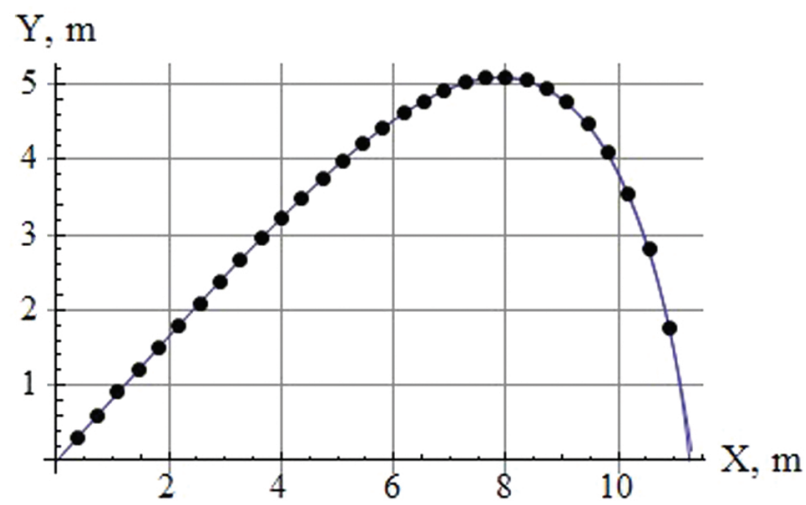

Figure 5: The trajectory of the shuttlecock.

Table 1: Basic parameters of the shuttlecock movement.

\begin{tabular}{llll}
\hline Parameter & Analytical & Numerical & Error \\
\hline & value & value & $(\%)$ \\
\hline$L,(\mathrm{~m})$ & 11.27 & 11.34 & -0.6 \\
\hline$H,(\mathrm{~m})$ & 5.10 & 5.06 & 0.8 \\
\hline$T,(\mathrm{~s})$ & 2.04 & 1.93 & 5.7 \\
\hline$x_{a},(\mathrm{~m})$ & 7.91 & 7.84 & 0.9 \\
\hline$t_{a},(\mathrm{~s})$ & 0.66 & 0.71 & -7.0 \\
\hline$\theta_{1}$ & $-82.2^{\circ}$ & $-79.4^{\circ}$ & 3.5 \\
\hline
\end{tabular}


air drag taken into account. All basic variables of the motion are described by analytical formulas containing elementary functions Moreover, numerical values of the sought variables are determined with high accuracy in a wide range of physical parameters. It can be implemented even on a standard calculator.

Of course, the proposed approach does not replace the direct numerical integration of the equations of the projectile motion, but only supplements it. The value and the advantage of the proposed formulas are that they replace a large number of approximate analytical solutions obtained previously by other authors. Thus, proposed formulas make it possible to study projectile motion with quadratic drag force even for first-year undergraduates.

\section{References}

[1] M. Turkyilmazoglu, European Journal of Physics 37, 035001 (2016).

[2] C. Belgacem, European Journal of Physics 35, 055025 (2014).

[3] A. Vial, European Journal of Physics 28, 657 (2007).

[4] G.W. Parker, American Journal of Physics 45, 606 (1977).

[5] K. Yabushita, M. Yamashita and K. Tsuboi, Journal of Physics A: Mathematical and Theoretical 40, 8403 (2007).

[6] P. Chudinov International Journal of Nonlinear Sciences and Numerical Simulation 3121 (2002)

[7] P. Chudinov Revista Brasileira de Ensino de Física 35, 1310 (2013).

[8] P. Chudinov, European Journal of Physics 25, 73 (2004).

[9] B.N. Okunev, Ballistics (Voyenizdat, İoscow, 1943).

[10] S. Timoshenko and D.H. Young, Advanced Dynamics (McGrow-Hill Book Company, New York, 1948). 